\title{
Age-related differences in adaptation during childhood: The influences of muscular power production and segmental energy flow caused by muscles
}

THOMAS KORFF ${ }^{1}$ AND JODY L. JENSEN ${ }^{2}$

${ }^{1}$ Brunel University

School of Sport and Education

Uxbridge, Middlesex

UB8 3PH

United Kingdom

Phone: +44 1985266477

Fax +44 1895269769

e-mail: thomas.korff@brunel.ac.uk

${ }^{2}$ The University of Texas at Austin

Department of Kinesiology \& Health

Austin, TX 78712

USA

Phone: +1 5122322685

e-mail: jlj@mail.utexas.edu

Acknowledgements.

This research was supported by the National Science Foundation under grant 998622. 


\section{Abstract}

Acquisition of skillfulness is not only characterized by a task-appropriate application of muscular forces but also by the ability to adapt performance to changing task demands. Previous research suggests that there is a different developmental schedule for adaptation at the kinematic compared to the neuro-muscular level. The purpose of this study was to determine how age-related differences in neuro-muscular organization affect the mechanical construction of pedaling at different levels of the task.

By quantifying the flow of segmental energy caused by muscles, we determined the muscular synergies that construct the movement outcome across movement speeds. Younger children (5-7 years; $n=11$ ), older children ( $8-10$ years; $n=8$ ), and adults ( $22-31$ years; $n=8$ ) rode a stationary ergometer at 5 discrete cadences ( $60 \mathrm{rpm}, 75 \mathrm{rpm}, 90 \mathrm{rpm}, 105 \mathrm{rpm}$, and $120 \mathrm{rpm}$ ). at 10\% of their individually predicted peak power output. Using a forward dynamics simulation, we determined the muscular contributions to crank power, as well as muscular power delivered to the crank directly and indirectly (through energy absorption) during the downstroke and the upstroke of the crank cycle.

We found significant Age x Cadence interactions for

- peak muscular power at the hip joint (Wilks' Lambda=.441, $\mathrm{F}(8,42)=2.65, \mathrm{p}=.019$ ) indicating that at high movement speeds children produced less peak power at the hip than adults

- muscular power delivered to the crank during the downstroke and the upstroke of the crank cycle (Wilks' Lambda=.399, $\mathrm{F}(8,42)=3.07$, $\mathrm{p}=.009$ ) indicating that children delivered a greater proportion of the power to the crank during the upstroke when compared to adults.

- $\quad$ hip power contribution to limb power Wilks' Lambda $=.454, \mathrm{~F}(8,42)=2.54, \mathrm{p}=.023)$ indicating a cadence-dependence of age-related differences in the muscular synergy between hip extensors and plantarflexors.

The results demonstrate that in spite of a successful performance, children construct the task of pedaling differently when compared to adults, especially when they are pushed to their performance limits. The weaker synergy between hip extensors and plantarflexors suggests that a lack of inter-muscular coordination, rather than muscular power production per-se, is a factor that limits children's performance ranges. 


\section{INTRODUCTION}

Successful movement is characterized by a task-appropriate application of muscular forces that results in desired limb trajectories. Skillfulness in movement incorporates, the ability to meet the demands of a particular task and the ability to perform the task over a range of task demands (Jensen, 2005). The reason for this is that for many tasks, the context in which they are performed changes constantly, necessitating adaptations by the neuro-motor system to accommodate the new task requirements. These adaptations are made by adjusting muscular forces. A common situation during which such muscular adjustments are made is present when the performer voluntarily changes certain parameters of the task.

It is empirically evident that the emerging capacity for voluntary adaptations of skill is a developmental phenomenon. For example, the range of speeds at which children can successfully perform cyclic tasks, such as walking or pedaling, is smaller than that of adults (Chao et al. 2002; Jeng et al. 1997; Liu et al. 2003). Chao et al. (2002) found children between 4 and 11 years of age to be less successful than adults as movement speed was increased from 40 to $120 \mathrm{rpm}$. Further, this effect was more pronounced in younger, compared to older, children.

In addition to behavioral differences, differences in neuro-muscular adaptive responses have also been observed. From the adult literature, we know that experienced cyclists activate their muscles earlier in the crank cycle when movement speed increases. There is a linear relationship between onsets of muscle activity and movement speed (Neptune et al. 1997). Chao et al. (2002) expanded on these results and determined age-related differences in the relationship between muscle onsets and movement speed. Their results showed that children (younger than 7 years of age) are less likely to demonstrate a linear relationship between muscle onsets and movement speed when compared to older children or adults. It is interesting to note that these neuro-muscular differences existed despite successful performance of the task, where successful performance was defined as pedaling within a certain range of the required target cadence. Bearing in mind the redundancy of the human system (Bernstein, 1967), the 
results by Chao et al. (2002) imply that -in spite of an observed adult-like task outcome- children's underlying neuro-muscular organization may be immature.

As children grow older and both their motor behavior and neuro-muscular organization improve, the following question remains unanswered: How do neuro-muscular differences relate to the outcome of the task? From differences in muscle activation patterns or muscular torque profiles alone (Chao et al. 2002), it is difficult to make inferences about the effect of such differences on the achievement of the task. To interpret these differences, we need to know the relationship between the muscular forces or torques and the kinematic outcome that they produce. Thus, the purpose of this study was to determine how agerelated differences in neuro-muscular organization affect the mechanical construction of pedaling at different levels of the task. In this investigation pedaling was chosen as the task to be studied because -in contrast to non-contact tasks- we have tight control over the movement outcome. Thus, we can specifically describe age-related differences in the neuro-muscular mechanisms underlying a kinematically well-defined task.

The first hypothesis tested was that children would differ from adults in the adjustment of peak muscular joint powers when they change movement speed: At low and moderate movement speeds, peak muscular joint powers (relative to the external power output) are predicted to be similar in children and adults; at high movement speeds, relative peak muscular joint powers are predicted to be smaller in children than in adults (hypothesis 1). To provide the rationale for this hypothesis, we start with the notion that maximum mechanical power (normalized to body mass or lean thigh volume) during pedaling is smaller in children, when compared to adults (Martin et al. 2000). Mechanical power (i.e., the overall power delivered to the crank) is composed of individual joint powers acting in synergy (Fregly and Zajac 1996). A possible explanation for the findings of Martin et al. (2000) is that the smaller observed normalized power in children is due to a reduced capacity to produce muscular power at the individual joints. Knowing that not only maximum power but also maximum movement speed is smaller in children than in adults (Chao et al. 2002; Liu et al. 2003), we hypothesized that in spite of a successful performance of the task - children would produce smaller 
relative peak muscular joint powers than adults when movement speed approaches their performance limits. Thus, hypothesis 1 was posed to test an Age x Cadence interaction.

The second hypothesis tested was that children would demonstrate a different distribution of muscular power throughout the crank cycle. Experienced adults deliver the majority of muscular power to the crank during the downstroke (0$180^{\circ}$ of the crank cycle) (Coyle et al. 1991; Fregly and Zajac 1996; Neptune et al. 2000). As peak power occurs during this region of the crank cycle, we hypothesized that the smaller relative peak joint powers, at high speeds in children would be accompanied by relatively less muscular power delivery to the crank when compared to adults (hypothesis 2). A corollary to this hypothesis is that children would compensate for this smaller relative power by delivering more relative muscular power to the crank than adults during the upstroke $\left(180-360^{\circ}\right.$ of the crank cycle). The reason for this is that muscular power delivered to the crank across the crank cycle needs to equal the external power output for each participant. Thus, if power is smaller in children during the downstroke, it needs to be compensated for by a greater amount of power during the upstroke.

A second corollary to hypothesis 2 is that the age-related differences in relative muscular power are accompanied by a different mechanical construction of the task. In pedaling, two mechanisms result in muscular power generation to the crank. Mechanical power can be generated by muscles to the crank directly or indirectly. In adult cyclists, the knee extensors act independently and deliver a large amount of muscular energy to the crank directly (Fregly and Zajac 1996; Neptune et al. 2000). The second mechanism that is responsible for muscular energy delivery to the crank is an indirect transfer of muscular energy. In adults, the proximal hip extensors deliver muscular energy to the limbs. A large amount of this energy is absorbed from the limbs by the plantarflexors and transferred to the crank indirectly (Fregly and Zajac 1996; Neptune et al. 2000). This mechanism is illustrated in Figure 1. In the Figure 1B, it can be seen that the net power produced at the hip joint (solid line) is almost identical to the hip power contribution to limb power (dotted line). This indicates that muscular energy produced by the hip extensors is almost exclusively delivered to the limbs. In 
Figure 1D, it can be seen that the ankle power contribution to limb power is negative between 0 and 180 degrees of the crank cycle. This indicates, that during this phase, mechanical energy is absorbed by the plantarflexors and transferred to the crank. As a consequence, the ankle power contribution to crank power (punctuated line) is greater than the net ankle power. Thus, at the ankle joint, a large amount of mechanical energy is transferred to the crank indirectly (through energy absorption from the limbs). This interplay between hip extensors and plantarflexors has been described as a muscular synergy between these two muscle groups (Fregly and Zajac 1996). In the remainder of this paper, the use of the term "muscular synergy" refers to this mechanical coupling between hip extensors and plantarflexors.

\section{Figure 1}

In our developmental context, the hypothesized smaller amount of relative muscular power at high speeds during the downstroke in children could have two possible explanations: (a) a smaller amount of direct muscular energy generation or (b) a smaller amount of indirect energy transfer (a weaker inter-muscular synergy). Similarly, the hypothesized compensatory increase in muscular power during the upstroke could be due to (a) an increase in direct muscular energy generation or (b) an increase in indirect energy transfer (a stronger inter-muscular synergy). Therefore we hypothesized an age-specific distribution of muscular power delivered to the crank directly and indirectly during the downstroke (hypothesis 3a) and during the upstroke (hypothesis 3b). One the one hand, one could expect that children use an increased indirect muscular energy transfer to the crank through the muscular synergy between the proximal and distal muscle groups (Neptune et al. 2000). On the other hand one could expect an increased direct muscular energy delivery to the crank. The fact that children's neuromuscular synergies are not fully developed at 10 years of age (Chao et al. 2002; Shumway-Cook and Woollacott 1985) provides support for this second possibility. 


\section{METHODS}

\section{Experimental Design}

Three groups of participants were recruited: a) younger children (YC, $\mathrm{n}=11,6.0 \pm$ 0.7 years of age); $b$ ) older children (OC, $n=8,9.4 \pm 0.9$ years of age); and c) adults ( $\mathrm{AD}, \mathrm{n}=8,27.3 \pm 2.3$ years of age). The inclusion of both $\mathrm{YC}$ and $\mathrm{OC}$ age groups allowed for a description of age-related changes in muscular force application between the ages of 5 and 10 years of age. Separating the age groups into children younger and older than 7 years of age allowed for the testing of age-related changes in muscular force application during a developmental period in which significant improvements in cycling performance and neuro-muscular adaptability are achieved (Chao et al. 2002; Jensen and Korff 2004; Liu et al. 2003; ShumwayCook and Woollacott 1985). The adult participants were used as a comparison reference, representing mature performance of the task. This was appropriate because it was our goal to attribute age-related differences in motor behavior to features of the neuro-motor system which can be assumed to be mature at 20 years of age. At the same time, we made sure that observed differences were not confounded by differences in the experience levels between performers.

All participants had moderate cycling experience. We interviewed the participants and the parent(s) to ensure that each participant knew how to ride a bicycle, but had no extensive bicycle riding experience. In particular, none of the participants had competed or participated in organized rides or races.

\section{Table 1}

\section{Fitting the Participant to the Bicycle}

Using a custom-made crank length adapter, the crank length was adjusted so that it approximated $20 \%$ of the participant's leg length. Leg length was defined as the distance between the greater trochanter and the sole of the foot during a standing position. The seat height was adjusted so that the relative knee angles at top dead center (TDC $-360^{\circ}$ of the crank cycle) and bottom dead center (BDC $-180^{\circ}$ of the crank cycle) were $75^{\circ} \pm 3^{\circ}$ and $155^{\circ} \pm 3^{\circ}$, respectively. The handlebars were adjusted so that the angle between the trunk and the horizontal axis of the inertial 
reference frame was $60^{\circ}$. The feet securely placed into toe-cages that were positioned on the pedal. These toe cages allowed for adjustments of the foot position in the anterior-posterior direction. For each participant, the position of the feet was adjusted so that the ball of the foot (metatarsal-phalangeal joint) was placed over the pedal spindle. For each participant and each condition, the resistance of the ergometer was set to $10 \%$ of predicted instantaneous peak power. Peak power was estimated from lean thigh volume using a method established by Martin et al. (2000).

\section{Procedure}

Participants rode a stationary ergometer (Monark, Model 829E) at 5 different speeds (60, 75, 90, 105, and $120 \mathrm{rpm})$ at $10 \%$ of their predicted peak power. Changes in resistance were achieved by adjusting the tension of a frictional belt surrounding the flywheel. To maximize the children's success rate, a blocked protocol was chosen over a random protocol. Participants started at a cadence of $60 \mathrm{rpm}$, which was then increased in increments of $15 \mathrm{rpm}$, up to $120 \mathrm{rpm}$. Each trial lasted $15 \mathrm{~s}$; the rest periods between trials lasted 20-60 s. The trial length and the rest periods were chosen to maximize performance success (defined as pedaling at the required target cadence), which was most critical in the youngest participants (Jensen and Korff 2004; Liu et al. 2003). The time span of the rest periods was chosen with the goal of obtaining the participants' full attention, which could result in longer rest periods for the children. For children, rest periods were extended to up to 5 minutes, if requested by the child. Visual and auditory feedback were given via a cycling computer and a metronome, in order to maximize the probability that the participants were pedaling at the required target cadence. During testing, the performance of the participants was monitored. If a participant did not hit the target cadence during a particular condition, he/she was allowed to repeat this condition after the regular testing protocol had been completed.

\section{Data Collection, Treatment, and Equipment}

Experimental data were collected with the goal to describe a biomechanical model of pedaling. The segments of each lower limb combined with the crank were modeled as 5-bar linkages (Fregly and Zajac 1996). Kinematic data were collected 
at $60 \mathrm{~Hz}$, using a 5-camera Vicon 250 system (Oxford Metrics, UK). Pedal forces were collected at $600 \mathrm{~Hz}$ by means of a custom-made pedal with two tri-axial piezoelectric force sensors (Kistler, model 9251AQ01). Kinematic data and force data were low-pass filtered with no phase lag at cutoff frequencies of $10 \mathrm{~Hz}$ and $20 \mathrm{~Hz}$ respectively, using Butterworth filters.

The joint centers were estimated from kinematic data. The center of the ankle joint was estimated from the coordinates of a marker placed on the lateral malleolus. The center of the knee joint was estimated from the coordinates of a marker placed on the lateral femoral epicondyle. The hip joint center was estimated from the coordinates of markers placed on the greater trochanter and the anterior superior iliac spine using a method described by Neptune and Hull (1995).

We used a gradient-based optimization algorithm (fminsearch, Mathworks Inc., MI) to find the joint positions that complied with the configuration constraints of the 5-bar linkage and differed minimally from those obtained from the kinematic data. During this optimization procedure, the segment lengths were allowed to deviate up to $5 \%$ from the calculated mean. Based on these optimal joint positions, the angular positions were calculated. Angular velocities and accelerations were obtained by fitting the position data to cubic splines and analytical differentiation.

The analyzed revolutions were chosen based on two inclusion criteria: First, the crank angular velocity, averaged over one crank cycle, had to be within \pm 5 rpm of the target cadence. Second, the averaged power produced by the right leg over one crank cycle had to be greater than $42.5 \%$ and smaller than $57.5 \%$ of the total power output. Ideally, the power produced by the ipsilateral leg would be $50 \%$ of the total power output. However, bilateral asymmetry leads to deviations from this ratio. The consequence is that the ipsilateral leg delivers more or less than $50 \%$ of the total power to the crank. To avoid a confounding effect on the dependent variables, a limit of acceptable bilateral asymmetry was defined. 
Before the data were analyzed, an analysis was performed to determine the sensitivity of the dependent variables to the effect of bilateral asymmetry. For this sensitivity analysis, the trial with the greatest accepted bilateral asymmetry observed in a member of the YC group at $60 \mathrm{rpm}$ - was chosen. For this trial, the reaction forces at the pedal were scaled so that the power produced by the ipsilateral leg was $50 \%, 42.5 \%$, and $57.5 \%$ of the external power output. The deviations of the dependent measures at the $42.5 \%$ and $57.5 \%$ conditions from those at the $50 \%$ condition were always smaller than $50 \%$ of one standard deviation of the mean in the $\mathrm{AD}$ group for the corresponding dependent variables. Therefore, the range of permitted bilateral asymmetry was considered acceptable and did not confound the analysis. The standard deviation of the AD group was chosen as a reference because in this group, the inter-subject variability was smallest for most of the dependent variables. Therefore, this standard can be considered conservative, emphasizing the negligibility of the possible confound of bilateral asymmetry in this study.

Depending on the number of revolutions within a trial that met the inclusion criteria, the kinematic and force data of up to 5 revolutions were averaged resulting in one representative revolution per participant and condition. This average revolution was then used for a forward dynamics simulation. The described inclusion criteria resulted in age group differences in the number of revolutions used. The mean values for included revolutions were 4.93, 3.63, and 2.76 for $\mathrm{AD}$, OC, and $\mathrm{YC}$ respectively.

For a total of 6 trials (5 in the YC group and 1 in the OC group), there were no revolutions that met the inclusion criteria. For 1 OC and 2 YC, no revolutions met the inclusion criteria at $105 \mathrm{rpm}$. This was also the case for $2 \mathrm{YC}$ at $60 \mathrm{rpm}$, and 1 YC at $120 \mathrm{rpm}$. These trials were eliminated from further analysis. The values for the dependent measures for these 6 trials were replaced by the group mean for the statistical analysis. Therefore, the minimum numbers of values contributing to a group mean of any dependent measure were 9 in the YC group. In the OC group, 7 values contributed to the group mean at $105 \mathrm{rpm}$, where 8 values contributed to the group means under the remaining conditions. 
The described age-related differences in cycling performance are consistent with previous findings (Chao et al., 2002; Jensen and Korff, 2004). By implementing the inclusion criteria, we analyzed the participants' best effort, and we were able to quantify age-related differences in the neuro-muscular mechanisms underlying behavior that was performed successfully.

\section{Forward Dynamics Simulation}

A forward dynamics simulation of cycling was used in this study. By using such a simulation, we can specifically quantify the flow of muscular energy (Fregly and Zajac 1996). Thus, it allows us to distinguish between muscular energy that is delivered to the crank directly and muscular energy that is delivered to the crank indirectly. This indirect energy transfer, achieved through energy generation to and energy absorption from the limbs, gives us information about the strength of intermuscular synergies.

A planar model of two-legged cycling actuated by muscle torques about the hip, knee, and ankle joints was developed (Fregly and Zajac 1996). The positions of the hip and the crank center of rotation were constrained to be fixed in space, and therefore the model consisted of two 5-bar linkages and possessed 3 degrees of freedom. The crank angle and the right and left hip angles were used as the independent degrees of freedom. The shank and foot angles and angular velocities were constrained to satisfy the kinematic constraint equations. The bicycle drive dynamics were modeled using an effective rotational resistive load and an effective rotational inertial load (Fregly et al. 2000).

All anthropometric parameters of the model were modified for each individual participant. The experimentally obtained values for body mass and segmental lengths were used. Segmental mass proportions, the center of mass locations, and moments of inertia were estimated using the regression equations presented by Jensen (1989). A feedback linearization algorithm (Seth et al. 2004) was used to find the tracking solution that resulted in the minimization of the differences between simulated and experimental data for each participant. A forward dynamics simulation was performed for each participant at each of the 5 cadences. 


\section{Dependent Variables}

Using the method described by Fregly and Zajac (1996), we quantified the following muscular power contributions:

- individual joint (ankle, knee, and hip) power contributions to crank power

- individual joint (ankle, knee, and hip) power contributions to limb power

- the summed contribution of the individual muscle torques of the right leg to crank power (muscular contribution to crank power)

All power profiles were normalized with respect to the average muscular contribution to crank power, in order to allow for meaningful comparisons between participants.

Regarding the hypothesis that at high speeds, peak muscular joint power would be lower in children compared to adults (hypothesis 1), the normalized peak powers at each joint were calculated. To test this hypothesis Age x Cadence, ANOVAs with repeated measures were performed for normalized peak muscular power at the hip, knee, and ankle joints.

To test the hypothesis that at high movement speeds, children would deliver relatively less relative muscular power to the crank than adults during the downstroke phase (hypothesis 2), the relative muscular contribution to crank power was averaged across the downstroke $\left(0^{\circ}-180^{\circ}\right)$ of the crank cycle, and an Age x Cadence ANOVA was performed.

To test the hypothesis that at high movement speeds, the amount of direct or indirect delivery of muscular energy to the crank during the downstroke and the upstroke would be different in children than in adults (hypotheses 3a and 3b), the following power contributions were averaged across both regions of the crank cycle: a) the relative contribution of hip power to limb power; b) the relative contribution of ankle power to limb power; and c) the relative direct contribution of knee power to crank power. Age x Cadence ANOVAs were performed for each of these power contributions during each region of the crank cycle. 
When the sphericity-assumption of an ANOVA was violated (Huynh-Feldt's $\varepsilon<0.75$ ), the multivariate method (Wilks' Lambda) was used (Schutz and Gessaroli 1987). In the case where Huynh-Feldt's $\varepsilon>0.75$, the univariate method was used and the degrees of freedom were adjusted accordingly. In cases where the Age x Cadence interaction was non-significant, we also tested that particular variable for an Age main effect, in order to determine if there were any age-related differences in the mechanical construction of the task - independent of cadence. In cases where the Age x Cadence interaction of an ANOVA was significant, follow up one-way ANOVAs with age being the between subject factor were performed on each cadence level for the corresponding dependent measure. If a follow-up ANOVA was significant, post-hoc t-tests (Student-Newman Keuls) were performed for pairwise comparisons between age group. In addiction, effect sizes (ES) were used to describe and interpret the pairwise comparisons (Cohen 1988). The type I error for all statistical analyses was .05.

\section{Tracking Experimental Data}

Before the statistical analyses were performed the tracking error was quantified by calculating the differences between the simulated and experimental data. This was done for angular positions, angular velocities and for horizontal and vertical force profiles. For each pair (simulated and experimental) of data profiles we calculated the relative absolute deviation (RAD - Equation 1).

$$
R A D=100 \cdot \frac{\frac{1}{n}\left[\sum_{i=1}^{n}\left|Y \exp _{i}-Y \operatorname{sim}_{i}\right|\right]}{\operatorname{range}(Y \exp )} \%
$$

where

$\operatorname{Yexp}_{\mathrm{i}}$ is the experimentally obtained data profile at the ith sample

$\mathrm{Ysim}_{\mathrm{i}}$ is the simulated data profile at the $\mathrm{i}^{\text {th }}$ sample

$\mathrm{n}$ is the number of samples for each profile

Averaged across all trials within each group, the tracking errors were $0.97 \%$ for each age group. 


\section{RESULTS}

\section{Peak Power - Hypothesis 1}

Age-related differences in relative peak power at the hip joint were dependent on cadence (Figure 2A). The hypothesized Age x Cadence interaction for relative peak power at the hip was significant (Wilks’ Lambda=.441, $F(8,42)=2.65$, $\mathrm{p}=.019)$. Follow up ANOVAs revealed that the effect of age on relative peak muscular power at the hip joint was significant at $105 \mathrm{rpm}$ and $120 \mathrm{rpm}(\mathrm{p}<.05)$. Post-hoc tests revealed that at $105 \mathrm{rpm}$, relative peak power at the hip for YC was significantly smaller than for AD ( $<<.05)$. No significant differences for pairwise comparisons were observed at $120 \mathrm{rpm}(\mathrm{p}>.05)$. The analysis of the effect sizes revealed that the direction of the age-group differences changed with increasing cadence. Although not significant, YC produced the largest values in relative peak power at the hip at 60 and $75 \mathrm{rpm}$ (the effect sizes being large or moderate). At 105 and $120 \mathrm{rpm}$, YC produced the smallest values in relative peak power (the effect sizes being large or moderate) (Table 2). At the knee and ankle joints, the Age $x$ Cadence interactions $(F(8,96)=0.75, \mathrm{p}=.640$ and $\mathrm{F}(8,96)=1.42, \mathrm{p}=.220$, respectively) and the main effects for age were non-significant $(F(2,24)=0.18$, $\mathrm{p}=.840$ and $\mathrm{F}(2,24)=1.10, \mathrm{p}=0.348$ ) (see Figures $2 \mathrm{~B}$ and $2 \mathrm{C}$, respectively).

\section{Figure 2}

\section{Table 2}

\section{Muscular Contribution to Crank Power - Hypothesis 2}

Age-related differences in the relative muscular contribution to crank power during the downstroke were dependent on cadence. The hypothesized Age x Cadence interaction was significant (Wilks’ Lambda=.399, $F(8,42)=3.07$, $\mathrm{p}=.009$ ). Follow up ANOVAs revealed that at all cadences, the age effect was statistically significant. At all cadences, the effect sizes (Table 3) describing the difference in the relative muscular contribution to crank power during the downstroke between $\mathrm{AD}$ and $\mathrm{YC}$ were large and positive, indicating that $\mathrm{AD}$ 
produced more relative muscular power during downstroke than YC (Figure 3a). Post-hoc tests revealed that at all cadences, the relative muscular contribution to crank power was significantly greater in $\mathrm{AD}$ when compared to $\mathrm{YC}(\mathrm{p}<.05)$. At 60 rpm and $105 \mathrm{rpm}$, the relative muscular contribution to crank power was greater in AD when compared to OC $(\mathrm{p}<.05)$. At all cadences below $120 \mathrm{rpm}$, the effect sizes describing the difference between $\mathrm{AD}$ and $\mathrm{OC}$ were large. Interestingly, only at $120 \mathrm{rpm}$ was the effect size moderate, indicating a considerably greater relative muscular power contribution to crank power in $\mathrm{AD}$ than $\mathrm{OC}$ only at cadences below $120 \mathrm{rpm}$. The effect sizes comparing OC and YC were small between 60 and $90 \mathrm{rpm}$ and large at 105 and $120 \mathrm{rpm}$. As all power profiles were normalized with respect to the average muscular crank power across the crank cycle, the described statistical effects and effect sizes were exactly reversed during the upstroke (Figure 3B). The profiles for the relative muscular contribution to crank power for all age groups and all cadences can be seen in Figure 4.

\section{Figure 3}

\section{Figure 4}

\section{Table 3}

\section{Muscular Synergy - Hypotheses $3 a$ and $3 b$}

\section{Hypothesis 3a-muscular synergy during the downstroke}

The smaller relative muscular contribution to crank power during the downstroke in children was accompanied by a weaker intermuscular synergy between hip extensors and plantarflexors. The Age x Cadence interaction for the relative hip power contribution to limb power was significant (Wilks’ Lambda=.454, $F(8,42)=2.54, p=.023)$. Follow up ANOVAs revealed that the age effect was only significant at $105 \mathrm{rpm}$ (Figure 5A). Post-hoc pairwise comparisons did not reveal any significant differences between the age groups ( $\mathrm{p}>.05)$. Although statistically not significant, the analysis of the effect sizes revealed that there was a cadencedependent reversal in the direction of the differences between children and adults. 
At the slow cadences ( $60 \mathrm{rpm}$ and $75 \mathrm{rpm}$ ) the effect sizes for the AD-OC and AD-YC comparisons were negative indicating that at these cadences, the relative hip power contribution to limb power was greater in children than in adults (see Figure 5A and Table 4). At the fast cadences (90 rpm - $120 \mathrm{rpm}$ ), the effect sizes for these comparisons were positive, indicating that at these cadences, the relative hip power contribution to limb power was greater in adults than in children. While the effect sizes for the AD-OC comparison were small at all cadences, the magnitude of the effect size was cadence-dependent for the AD-YC comparison. For this comparison, the effect sizes were small at the slow cadences and moderate or large the high cadences. For the OC-YC comparison, all effect sizes were small at all cadences except for $105 \mathrm{rpm}$.

The Age $\mathrm{x}$ Cadence interaction for the relative ankle power contribution to limb power was significant (Wilks' Lambda=.334, $\mathrm{F}(8,42)=3.83$, $\mathrm{p}=.002$ ). Follow up ANOVAs revealed that the age effect was significant at $60 \mathrm{rpm}, 90 \mathrm{rpm}$, and 105 rpm $(\mathrm{p}<.05)$. At these cadences, the relative ankle power contribution to limb power was significantly smaller (more negative) in AD than in YC. In addition at $105 \mathrm{rpm}$, the ankle power contribution to limb power was significantly smaller in AD when compared to OC $(\mathrm{p}<.05)$. The effect sizes showed that the direction for all pairwise comparisons was consistent across all cadences (Table 4). AD’s relative ankle power contribution to limb power was smaller (more negative) than that of OC or YC, and OC's relative ankle power contribution to limb power was smaller than that of YC (Figure 5B). For the AD-YC comparison, the effect sizes were large at all cadences (Table 4). Although the direction for all pairwise comparisons was consistent, the trend with regards to the magnitude of the effect sizes was not consistent for the AD-OC and OC-YC comparisons across cadences.

\section{Figure 5Table 4}

\section{Hypothesis $3 b-$ muscular synergy during the upstroke}

Children compensated for the relative smaller muscular contribution to crank power during the downstroke by producing a larger relative knee power contribution to crank power during the upstroke. For the relative hip and ankle power contributions to limb power during the upstroke, the Age x Cadence 
interactions failed statistical significance (Wilks' Lambda $=.713, \mathrm{~F}(8,42)=0.97$, $\mathrm{p}=.474$ for the hip and Wilks' Lambda=.543, $\mathrm{F}(8,42)=1.87, \mathrm{p}=.090$. for the ankle). The main effects for age for these measures were also non-significant $(F(2,24)=0.23, p=.794$ for the hip and $F(2,24)=2.96, p=.071$ for the ankle) .

In contrast, the direct knee power contribution to crank power was greater in children than in adults (Figure 6). Although the Age x Cadence interaction was non-significant (Wilks' Lambda $=.581, \mathrm{~F}(8,42)=1.64$, $\mathrm{p}=1.43$ ), the main effect for Age for this measure during the upstroke was significant $(F(2,24)=6.27, \mathrm{p}=.006)$. Post-hoc tests revealed that the direct knee power contribution to crank power was significantly greater in $\mathrm{YC}$ and $\mathrm{OC}$ when compared to $\mathrm{AD}(\mathrm{p}<.05)$. The effect sizes describing the AD-OC and AD-YC comparisons (collapsed across all cadences) were large (ES=-1.29 and ES=-1.71, respectively), while the effect size describing the OC-YC comparison was small (ES=-0.23).

\section{Figure 6}




\section{DISCUSSION}

The results of the present investigation demonstrate that the neuro-muscular mechanism which children use to voluntarily adapt to changes in movement speed is different compared to adults. At high movement speeds, children demonstrated smaller muscular joint powers at the hip joint which were accompanied by a weaker muscular synergy during the downstroke of the crank cycle when compared to adults. Children compensated by delivering more muscular energy directly to the crank during the upstroke of the crank cycle.

The first hypothesis was posed to test if at high movement speeds, children, compared to adults, would produce less maximum muscular power at the ankle, knee, and hip joints. This hypothesized relationship was only present at the hip joint indicating a joint-dependence of this effect. In conformity with hypotheses 2 and 3a, the smaller relative peak muscular joint power was accompanied by a smaller relative muscular power delivered to the crank during the downstroke and a weaker synergy between hip extensors and plantarflexors. Hypotheses 3b was posed to determine how children would compensate for the smaller relative muscular power delivery to the crank during the downstroke when compared to adults. Children demonstrated greater relative muscular power delivery to the crank during the upstroke by using their knee flexors to deliver more muscular energy directly to the crank.

The age-related differences in the mechanical construction of the pedaling task were most apparent in the younger children. They demonstrated a weaker synergy between hip extensors and plantarflexors at high movement speeds during the downstroke than older children and adults. It is important to note that in YC, energy was absorbed from (and not delivered to) the limbs by the hip extensors at high movement speeds. Thus, in these children, the hip extensor-plantarflexor synergy was completely missing during this phase, and they did not take any advantage of this synergy to facilitate energy delivery to the crank at high movement speeds. 
The averaged normalized muscular power delivery to the crank across the crank cycle was equal for all participants. Thus, children needed to compensate for the reduced muscular power production during the downstroke by a greater relative muscular power production during the upstroke. This greater magnitude in relative muscular power was achieved by more direct relative muscular energy delivery by the knee flexors to the crank. We can conclude that, children compensated for their weaker inter-muscular synergy by producing a greater amount of direct muscular energy delivery to the crank.

Although our results suggest that children compensated for reduced muscular power production during the downstroke by increasing direct knee power contributions to crank power, it cannot be fully ruled out that indirect energy transfer was also used as a compensatory mechanism. Although no age-related differences in the ankle power contribution to limb power were found during the upstroke, it is possible that differences in the contributions of individual muscles exist. Neptune et al. (2000) demonstrated that during parts of the upstroke, the plantarflexors and dorsiflexors are co-active and have opposite effects. While the dorsiflexors absorb energy from the limbs, the plantarflexors deliver energy to the limbs. This mechanism cannot be revealed in an analysis of net muscular power at the ankle which is a limitation of the torque driven model.

The findings of the present investigation have important implications for teachers, coaches, and therapists, because they suggest that it is a lack of intermuscular coordination rather than muscular power production per se that limits the range of movement speeds at which children can perform the task successfully. A limitation to this speculation is the fact that we analyzed only successful trials. Therefore, it is possible that children chose to construct the task differently at high movement speeds. However, previous research has shown that $120 \mathrm{rpm}$ is close to children's performance limits (Chao et al. 2002; Liu et al. 2003), and we can assume that scaling up the task close to this limit would reduce the number of possible solutions of the task sufficiently to evoke the observed behavior.

The results of this investigation confirm and extend previous findings about differences in voluntary adaptive skill performance between children younger and 
older than 7 years of age. In OC, relative peak power at the hip was considerably greater when compared to YC. Differences in relative muscular power delivery to the crank between these two age groups were only present at the two highest cadences. Interestingly, differences between OC and AD tended to decrease with increasing cadence, while differences between OC and YC tended to increase with increasing cadence. Together these findings demonstrate an emerging adult-like mechanism underlying the construction of pedaling during childhood. The fact that older children became more adult-like only at the higher cadences lets us speculate that an adult-like mechanical construction is necessary to successfully pedal at very high cadences (i.e. beyond $120 \mathrm{rpm}$ ), bearing in mind that older children tend to be more successful than younger children at pedaling at high cadences (Chao et al., 2002). Though not specifically tested, we can infer from the observed reduction in muscular power generation at the hip and the weaker hip extensor-plantarflexor synergy is a contributor to the reduced rate of children's success at high movement speeds (Chao et al. 2002). However, the data do not provide incontrovertible support for this speculation, because only successful revolutions were analyzed.

Our results stand in agreement with previous findings that there are significant changes in the neuro-muscular synergies between the ages of 6 and 9 years of age (Chao et al. 2002). Chao et al. (2002) demonstrated that children older than 7 years of age are more likely to show an organized response in terms of muscle activation patterns in response to cadence changes in pedaling than children younger than 7 years of age. The degree of organization of the muscle response was defined as the strength of the relationship between movement speed and onsets of muscle activity. Both age groups had the same level of bicycle riding experience, thus the observed differences were age-related and not experiencerelated. The results of the present investigation confirm these results and extend them by an explicit attribution of the observed differences between younger and older children to age-related differences in the production of hip power and the muscular energy delivery to the crank. This is an important step toward understanding the mechanisms that lead to previously observed differences in muscle activation patterns between 4 and 10 years of age (Chao et al. 2002). 
Another possible source for the weaker synergy between hip extensors and plantarflexors is the children's inability to sufficiently stiffen the ankle joint to allow for an efficient transfer of muscular energy from the limbs to the crank. However, the fact that no age-related differences in peak power at the ankle joint were found suggests that children were able to generate sufficient muscular power at the ankle joint, even at high movement speeds. A limitation to this conclusion is that differences in the degree of co-contraction of plantarflexors and dorsiflexors could also contribute to age-related differences in ankle joint stiffness. Future research should be aimed at investigating the reduced hip extensor-plantarflexor synergy on a muscular level and at specifically attributing this reduced synergy to children's failure in performance at high movement speeds.

In addition to the cadence-dependent age effects, we found age-related differences in the construction of the task that were independent of cadence. Our results demonstrate that children produce relatively more muscular power during the upstroke when compared to adults - independent of cadence. This suggests that children construct the task of pedaling differently at their preferred cadences and not only if they are pushed to their performance limit. Coyle et al. (1991) provide a possible explanation for this finding. These authors found that elite cyclists produce a greater proportion of the propulsive force applied to the crank during the downstroke when compared to sub-elite cyclists. They suggest that elite cyclists may be able to recruit a greater proportion of muscle with each revolution. Expanding on this argumentation and in the light of our results, we can speculate that children are not able to recruit a sufficient proportion of muscle during the downstroke in order to demonstrate an adult-like force production.

Finally, the results of the present investigation extend our knowledge about the relationship between adaptation on a kinematic and a neuro-muscular level. Jensen and Korff (2004) investigated children's response to voluntary changes in movement speed in terms of kinematic variability. Their results indicate that at moderate and moderately high speeds (60 rpm-100 rpm), children between 4 and 14 years of age adapt in an adult-like fashion. Our results extend the findings by Jensen and Korff (2004) by including kinetic variables making possible inferences about neuro-muscular synergies, while Jensen and Korff (2004) investigated 
adaptability only on a kinematic level (i.e., in terms of kinematics). It is of particular significance that differences in the neuro-muscular synergies exist although the task is performed successfully. By using cycling -a closed chain contact task- and by adjusting the position of each participant in the ergometer, we constrained the kinematics of the behavior. In addition, we only analyzed revolutions which were within a close range of the target cadence. By closely controlling the kinematics of the behavior, we demonstrated that the analysis on a kinematic level does not necessarily reveal neuro-muscular mechanisms that lead to differences (or similarities) in the movement outcome. This notion has implications for teachers, coaches, and therapists, who are most often limited to an analysis of observable movement.

\section{Summary}

In summary, the results of this study revealed age-related differences in voluntary adaptive skill in terms of muscular power production. At high movement speeds, relative maximum muscular power at the hip joint was smaller in children when compared to adults. During the downstroke, a smaller amount of indirect energy transfer was observed in children when compared to adults. Children compensated for this by delivering more muscular energy directly to the crank during the upstroke. These results suggest that it is the ability to efficiently take advantage of the transfer of segmental energy (the strength of the muscular synergy) that leads to age-related differences in voluntary adaptation, rather than a reduction in muscular power generation per se. Children compensated for the lack of intermuscular synergies by direct muscular energy delivery to the crank using their knee flexors which is possibly energetically more costly.

\section{Implications}

The results extend our knowledge by describing children's capacity to adapt over a broad range of task demands and by attributing observed overall differences to a specific muscular synergy. These findings are important because they increase our knowledge about factors that lead to age-related improvement in adaptive skills and give us hints about possible factors that limit the range of task demands to which children can adapt. Future research should be aimed at using this information to determine the factors that lead to performance failure at high movement speeds, and to further illuminate the source of the observed 
differences. In particular, it is of interest to ascertain why younger children demonstrate a reduction in hip joint power. Possible explanations are differences in muscle-intrinsic properties (Asai and Aoki 1996; Lexell et al. 1992) or in recruitment strategies (Gibbs et al. 1997). Musculo-skeletal modeling techniques will be helpful in answering these questions. 


\section{References}

Asai H, Aoki J (1996) Force development of dynamic and static contractions in children and adults. International Journal of Sports Medicine 17:170-174

Bernstein N (1967). Coordination and regulation of movement. Pergamon Press, New York

5 Chao P, Rabago C, Korff T, Jensen JL (2002). Muscle activation adaptations in children in response to changes in cycling cadence [Abstract]. Journal of Sport Exercise Psychology 24(Suppl.):S42-S43

Coyle EF, Feltner ME, Kautz SA, Hamilton MT, Montain SJ, Baylor AM, Abraham LD, Petrek GW (1990). Physiological and biomechanical factors associated with elite endurance cycling performance. Medicine and Science in Sports and Exercise 23:93-107.

10 Fregly BJ, Zajac FE (1996). A state-space analysis of mechanical energy generation absorption and transfer during pedaling. Journal of Biomechanics 29:81-90

Fregly BJ, Zajac FE, Dairaghi CA (2000). Bicycle drive system dynamics: theory and experimental validation. Journal of Biomechanical Engineering 122:446-452

Gibbs J, Harrison LM, Stephens JA (1997). Cross-correlation analysis of motor unit activity recorded from

15 two separate thumb muscles during development in man. Journal of Physiology 499:255-266

Jacobs R, van Ingen Schenau GJ (1992). Control of an external force in leg extensions in humans. Journal of Physiology 457:611-626

Jeng SF, Liao HF, Lai JS, Hou JW (1997). Optimization of walking in children. Medicine and Science in Sports and Exercise 29:370-376

20 Jensen JL, Korff T (2004). Adapting to changing task demands: variability in children’s response to manipulations of resistance and cadence during pedaling. Research Quarterly for Exercise and Sport 75:361369

Jensen JL (2005). The puzzles of motor development: how the study of developmental biomechanics contributes to the puzzle solutions. Infant and Child Development, 14: 501-511.

25 Jensen RK (1989). Changes in segment inertia proportions between 4 and 20 years. Journal of Biomechanics 22:529-536

Kane TR, Levinson DA (1985). Dynamics: Theory and Applications. McGraw-Hill Book Company, New York

Lexell J, Sjostrom M, Nordlund AS, Taylor CC (1992). Growth and development of human muscle: A

30 quantitative morphological study of whole vastus lateralis from childhood to adult age. Muscle Nerve 15:404409

Liu T, Korff T, Chao P, Jensen JL (2003). Bilateral asymmetry and cycling performance in children.

[Abstract]. Journal of Sport Exercise Psychology 25(Suppl.):S91

Martin JC, Farrar RP, Wagner BM, Spirduso WW (2000). Maximal power across the lifespan. Journal of

35 Gerontology: Medical Sciences 55A:M311-M316

Neptune RR, Hull ML (1995). Accuracy assessment of methods for determining hip movement in seated cycling. Journal of Biomechanics 28:423-437

Neptune RR, Herzog W (1999). The association between negative muscle work and pedaling rate. Journal of Biomechanics 32:1021-1026

40 Neptune RR, Kautz SA, Zajac FE (2000). Muscle contributions to specific biomechanical functions do not change in forward versus backward pedaling. Journal of Biomechanics 33:155-164

Raasch CC, Zajac FE, Ma B, Levine WS (1997). Muscle coordination of maximum-speed pedaling. Journal of Biomechanics 30:595-602 
Seth A, McPhee JJ, Pandy MG (2004). Multi-joint coordination of vertical arm movement. Applied Bionics and Biomechanics 1:45-56

Shumway-Cook A, Woollacott MH (1985). The growth of stability: Postural control from a developmental perspective. Journal of Motor Behavior 17:131-147

5 Yang JF, Stephens MJ, Vishram R (1998). Infant stepping: a method to study the sensory control of human walking. Journal of Physiology 507:927-937 


\section{Figure Captions}

Figure 1. Mechanical energy distribution due to all muscles of one side of the body (A), and torques at the hip (B), knee (C), and ankle (D) for an experienced adult cyclist. The net muscular power at each joint is decomposed into power contributions to the crank and the limbs. If the net power of a particular joint is positive, mechanical energy is added to the system. If it is negative, then energy is absorbed. If the crank or limb power contributions are positive, energy is delivered to the crank or the limbs, respectively. If these contributions are negative, energy is absorbed from the crank or the limbs, respectively. These data were obtained in our lab from an experienced cyclist at $75 \mathrm{rpm}$ at $96 \mathrm{~W}$. All power profiles are on the same ordinate scale.

Figure 2. Effect of cadence on peak power at the ankle (A), knee (B), and hip (C) joints. The symbol “*” indicates a significant age-effect at the corresponding cadence. Means and standard deviations are plotted for adults (AD), older children (OC), and younger children (YC).

Figure 3. Effect of age and cadence on the relative muscular power contribution of the right limb to crank power during the downstroke (A) and during the upstroke (B). The symbol “*” indicates a significant age-effect at the corresponding cadence. Means and standard deviations are plotted for adults (AD), older children (OC), and younger children (YC).

Figure 4. Normalized muscular power contributions of the right limb to crank power for adults (AD), older children (OC), and younger children (YC) at 5 different cadences. The data for each age group are averaged across participants

Figure 5. Effect of age and cadence on the relative hip power contribution to limb power (A), the relative ankle power contribution to limb power (B). The symbol “*” indicates a significant ageeffect at the corresponding cadence. Means and standard deviations are plotted for adults (AD), older children (OC), and younger children (YC).

Figure 6. Effect of age on the relative knee power contribution to crank power during the upstroke. The symbol “*” indicates a statistically significant effect. Means (collapsed across cadences) and standard deviations are plotted for adults (AD), older children (OC), and younger children (YC). 


\section{Tables}

Table 1. Group characteristics of the participants who were included in the final analysis: Means \pm standard deviations are presented for age, predicted peak power, and bicycle riding experience.

\begin{tabular}{|c|c|c|c|c|}
\hline & N & \multicolumn{2}{|c|}{ Age (years) } & $\begin{array}{c}\text { Predicted } \\
\text { Peak Power } \\
\text { (W) }\end{array}$ \\
\cline { 3 - 4 } & & Range & Mean \pm SD & \\
\hline Younger Children (YC) & 11 & $5-7$ & $6.0 \pm 0.7$ & $256 \pm 30$ \\
\hline Older Children (OC) & 8 & $8-10$ & $9.4 \pm 0.9$ & $392 \pm 137$ \\
\hline Adults (AD) & 8 & $25-31$ & $27.3 \pm 2.3$ & $1015 \pm 258$ \\
\hline
\end{tabular}

Table 2. Effect sizes describing pairwise age group differences in the relative maximm hip joint power ( $\mathrm{AD}=$ =adults; $\mathrm{OC}=$ older children; $\mathrm{YC}=$ younger children).

\begin{tabular}{|c|c|c|c|c|c|}
\hline & \multicolumn{5}{|c|}{ Cadence (rpm) } \\
\hline Comparison & $\mathbf{6 0}$ & $\mathbf{7 5}$ & $\mathbf{9 0}$ & $\mathbf{1 0 5}$ & $\mathbf{1 2 0}$ \\
\hline AD-OC & -0.55 & -0.34 & 0.60 & 0.65 & 0.14 \\
\hline AD-YC & -0.93 & -0.76 & 0.39 & 1.15 & 1.54 \\
\hline OC-YC & -0.57 & -0.55 & -0.27 & 0.64 & 0.98 \\
\hline
\end{tabular}

Table 3. Effect sizes describing pairwise age group differences in the relative muscular power contribution to crank power ( $\mathrm{AD}=$ adults; $\mathrm{OC}=$ older children; $\mathrm{YC}=$ younger children).

\begin{tabular}{|c|c|c|c|c|c|}
\hline & \multicolumn{5}{|c|}{ Cadence (rpm) } \\
\hline Comparison & $\mathbf{6 0}$ & $\mathbf{7 5}$ & $\mathbf{9 0}$ & $\mathbf{1 0 5}$ & $\mathbf{1 2 0}$ \\
\hline AD-OC & 2.31 & 1.01 & 1.01 & 1.34 & 0.58 \\
\hline AD-YC & 1.47 & 1.19 & 1.35 & 1.90 & 1.73 \\
\hline OC-YC & -0.19 & 0.47 & 0.49 & 0.94 & 0.94 \\
\hline
\end{tabular}


Table 4. Effect sizes describing pairwise age group differences in the relative hip power contribution to limb power and the relative ankle power contribution to limb power ( $\mathrm{AD}=$ adults; OC=older children; YC=younger children).

\begin{tabular}{|c|c|c|c|c|c|}
\hline Comparison & \multicolumn{5}{|c|}{ Cadence } \\
\hline $\begin{array}{c}\text { Hip Power Contribution } \\
\text { to Limb Power }\end{array}$ & $\mathbf{6 0}$ & $\mathbf{7 5}$ & $\mathbf{9 0}$ & $\mathbf{1 0 5}$ & $\mathbf{1 2 0}$ \\
\hline AD-OC & -0.43 & -0.48 & 0.24 & 0.18 & 0.46 \\
\hline AD-YC & -0.32 & -0.24 & 0.50 & 1.10 & 0.70 \\
\hline OC-YC & -0.04 & 0.12 & 0.36 & 1.20 & -0.18 \\
\hline $\begin{array}{c}\text { Ankle Power } \\
\text { Contribution to Limb } \\
\text { Power }\end{array}$ & \multicolumn{5}{|c|}{} \\
\hline AD-OC & -1.11 & -0.25 & -0.82 & -1.12 & -0.64 \\
\hline AD-YC & -1.28 & -0.88 & -1.70 & -2.29 & -1.69 \\
\hline OC-YC & -0.31 & -0.65 & -0.69 & -1.15 & -0.33 \\
\hline
\end{tabular}

